\title{
Hand Written Recognition Using Modified N_Tuple Method
}

\author{
Fadhil Hanoon Abbood ${ }^{1}$ Intisar Abid Yousif ${ }^{2}$ Shaimaa Khudhair Salah $^{3}$ \\ Department of Computer Science, College of Education
}

Al Mustansiriyah University

Iraq

\begin{abstract}
In a world running on data, accurate handwriting recognition could become a powerful tool. With it, hastily scribbled notes and formal, handwritten letters become readable by a computer.

In a writer recognition system, the system searches "one to many in a wide database of manual samples from established authors, and returns a potential list of candidates. A n-tuple method of pattern recognition is developed and evaluated for use with unique references to unconstraint manually written characters for classification of non-deterministic content. A new method is seen to update the system and to increase the implementation and certainty of the findings. The results show that a superior can be achieved and show the legitimacy of the procedures proposed.
\end{abstract}

Key Words: Hand Written, $N \_T u p l e$, Discrete Wavelet Change (DWT).

\section{INTRODUCTION}

Artificial neural systems are computational models in light of the standards of the organic neural systems. The fundamental normal for the model is the presence of a straightforward preparing unit, called a neuron, with the capacity to play out a weighted entirety of its information sources, register its inside state as per this aggregate and for the situation that the state is over a limit send an excitatory flag to its yield. The capacity of the neural system depends on the systems administration of these essential components to frame a parallel and dispersed handling framework. Every association among the neurons can have an esteem deciding its quality, or weight. These qualities are balanced amid the preparation period of the system and it is in these weights where the data is encoded in the neural systems [1][2].The digital learning network embodies the $\mathrm{n}$-tuple pattern recognition system. In this process, several features, called n-tuples, are created from the input pixel matrix and are the basis for training and acknowledgement. These networks have no weighted associations of neuronal hubs (RAM units) and operate with paired sources and rates of knowledge. Random Access Memory (RAM) shows the neuronal capacity. The training process includes modifying the RAM contents directly, instead of manipulating the weight between nodes (RAMs).. This characteristic generally allows a faster learning than weighted neural networks, since by modifying weights the previously learned information is also modified. In the more flexible digital neural networks, the RAMs can learn new information by changing only the memory contents corresponding to the new input pattern, without modifying the information related to other inputs[1]. This research presents a method to enhance the classification of the n-tuple network depending on the enhancement process of the image Characteristics.

\section{STRUCTURE OF DIGITAL LEARNING NETWORKS (N_Tuple METHOD)}

Advanced Learning Networks depend on basic handling units. These units can remember input- yield combines these units is RAMs[3]. The advanced learning system process takes as its contribution from a paired picture. From this picture an arrangement of tuples are framed. Each tuple is comprised of $n$ components from the picture. The cause of every pixel in the picture used to make up each tuple is characterized once. Typically the cause of every pixel is chosen in irregular or requested way, every pixel just adding to one tuple. The items to be perceived as having a place with some class have certain properties that we use to recognize classes [1].

These properties (traits) are the recognizable, where the perception gives an incentive to every one of an arrangement of properties. A settled arrangement of properties is utilized for a specific populace and the arrangement of their esteems for a question decides if it has a place with a class or not. The individual properties are called highlights of the populace [4]. 
Assume that there are $\mathrm{n}$ highlights for a populace to be utilized for acknowledgment. These $\mathrm{n}$ highlights are requested into a $\mathrm{n}$ tuple with the goal that an arrangement of watched esteems for a question frames a vector, called an element vector . Along these lines, the component vectors speak to the articles in a populace. Example acknowledgment is done on include vectors. The ideal size of $\mathrm{n}$ relies upon the qualities of the information and the speculation properties [3].Learning in a RAM unit happens basically by composing into the comparing areas. This learning procedure is substantially less complex than the modification of weights. The RAM, as characterized above, can figure every twofold capacity of its info while the weighted-entirety and-limit hubs can just register straightly distinct capacities. There is no generalization in the RAM node itself. Generalization can be interpreted to provide the correct performance for trends which are not seen in the training process. RAM nodes not only generate the right output for the input patterns in the training stage. There is still widespread use of RAM units in networks [5-6].

The most straightforward RAM connect with properties of speculation is known as a discriminator and is appeared in figure 1. This comprises of a layer of $\mathrm{K}$ data sources and hence each RAM stores $2 \mathrm{n}$ one- piece words, and the single layer gets a twofold example of $\mathrm{K} . \mathrm{N}$ bits. It is accepted that, before any preparation happens, all the memory cells in the RAMs are set to 0 . Preparing comprises of applying a ' input design ' of 0 's and 1's at the information terminals. This called a preparation design and is a case of the class of examples to be learnt by the discriminator. To record this example, a 1 is put away in that memory area of each RAM, which is tended to by this info design. Viably, this makes each RAM record the event of part of the info design - the part 'examined' by that RAM. This is improved the situation other information designs prompting further 1's being put away in the RAM [3-7]. 


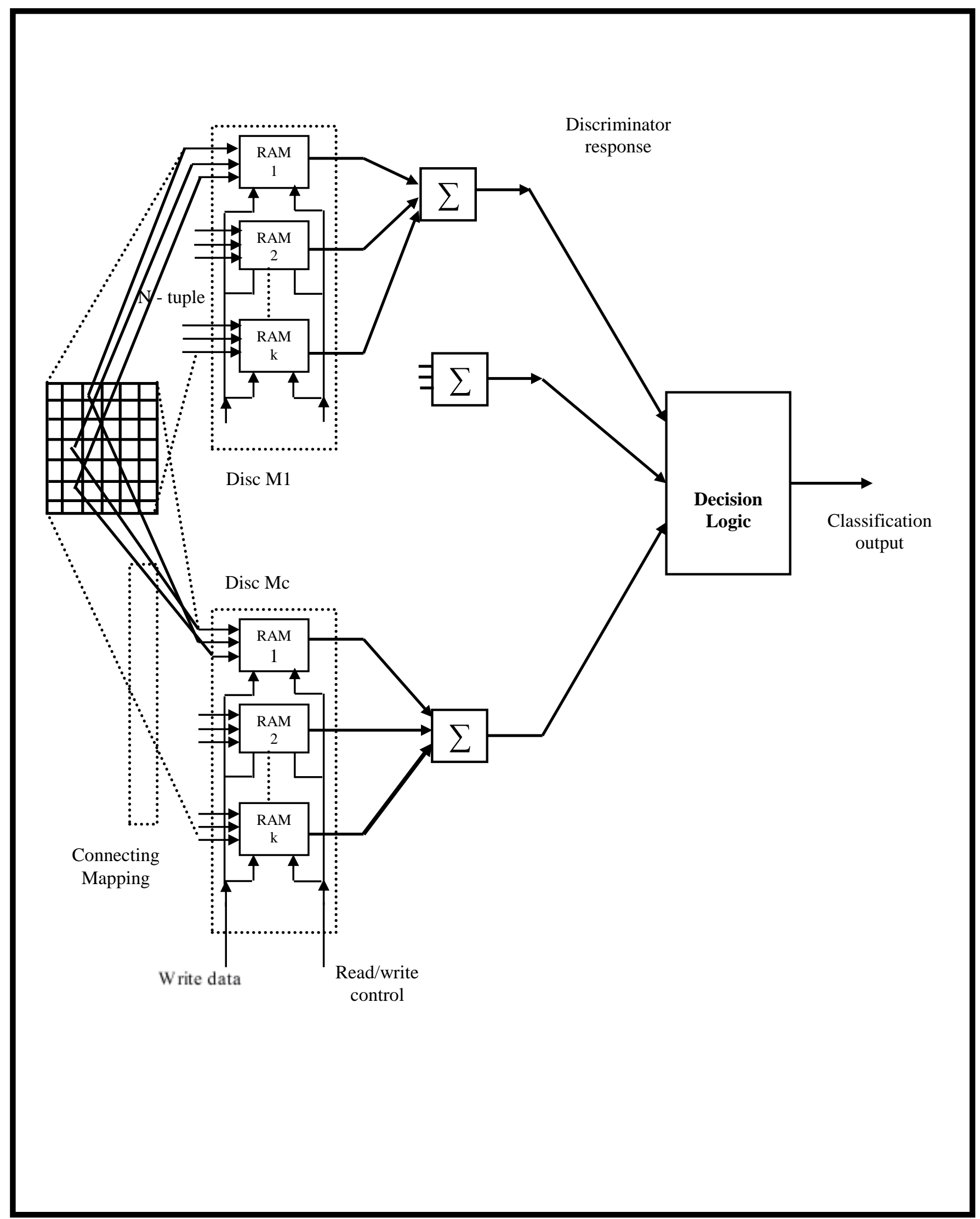

Figure 1: Structure of Digital Learning Networks (multi categories) 
International Journal of Advances in Scientific Research and Engineering (ijasre), Vol 6 (12), December -2020

\section{3 . An Example of a Digital Learning Networks}

This case represents the task of the rearranged acknowledgment gadget appeared in figure 2, utilizing a 3 x 3 input design. The planning collection includes four examples of streamlined letters ' $\mathrm{H}$ ' and 'L.' Two mutilated characters form the test set (Figure 2). In the wake of preparing the RAM units have the accompanying filling:

\begin{tabular}{|c|c|c|c|c|c|c|c|c|}
\hline \multicolumn{9}{|c|}{ Tuple $=$ Address } \\
\hline & 000 & 001 & 010 & 011 & 100 & 101 & 110 & 111 \\
\hline$R A M 11$ & 0 & 0 & 0 & 0 & 1 & 1 & 0 & 0 \\
\hline$R A M 12$ & 0 & 0 & 0 & 0 & 0 & 0 & 0 & 1 \\
\hline$R A M 13$ & 0 & 0 & 0 & 0 & 0 & 1 & 0 & 0 \\
\hline$R A M 21$ & 0 & 0 & 0 & 0 & 1 & 0 & 0 & 0 \\
\hline$R A M 22$ & 0 & 0 & 0 & 0 & 1 & 0 & 0 & 0 \\
\hline$R A M 23$ & 0 & 0 & 0 & 0 & 1 & 0 & 0 & 1 \\
\hline
\end{tabular}

The corresponding reaction is given in case of a test design being produced for the system: For test design 1 , the $\mathrm{H}-$ discriminator gives ' 1 ' and the $\mathrm{L}$-discriminator gives a '2.' The $\mathrm{H}$ - discriminator gives a ' 2 ' for the second test designs and the $\mathrm{L}-$ discriminator gives a ' 0 .' If the edge is set to two, the ' $\mathrm{L}$ ' and ' $\mathrm{H}$ ' are both interpreted precisely [3].

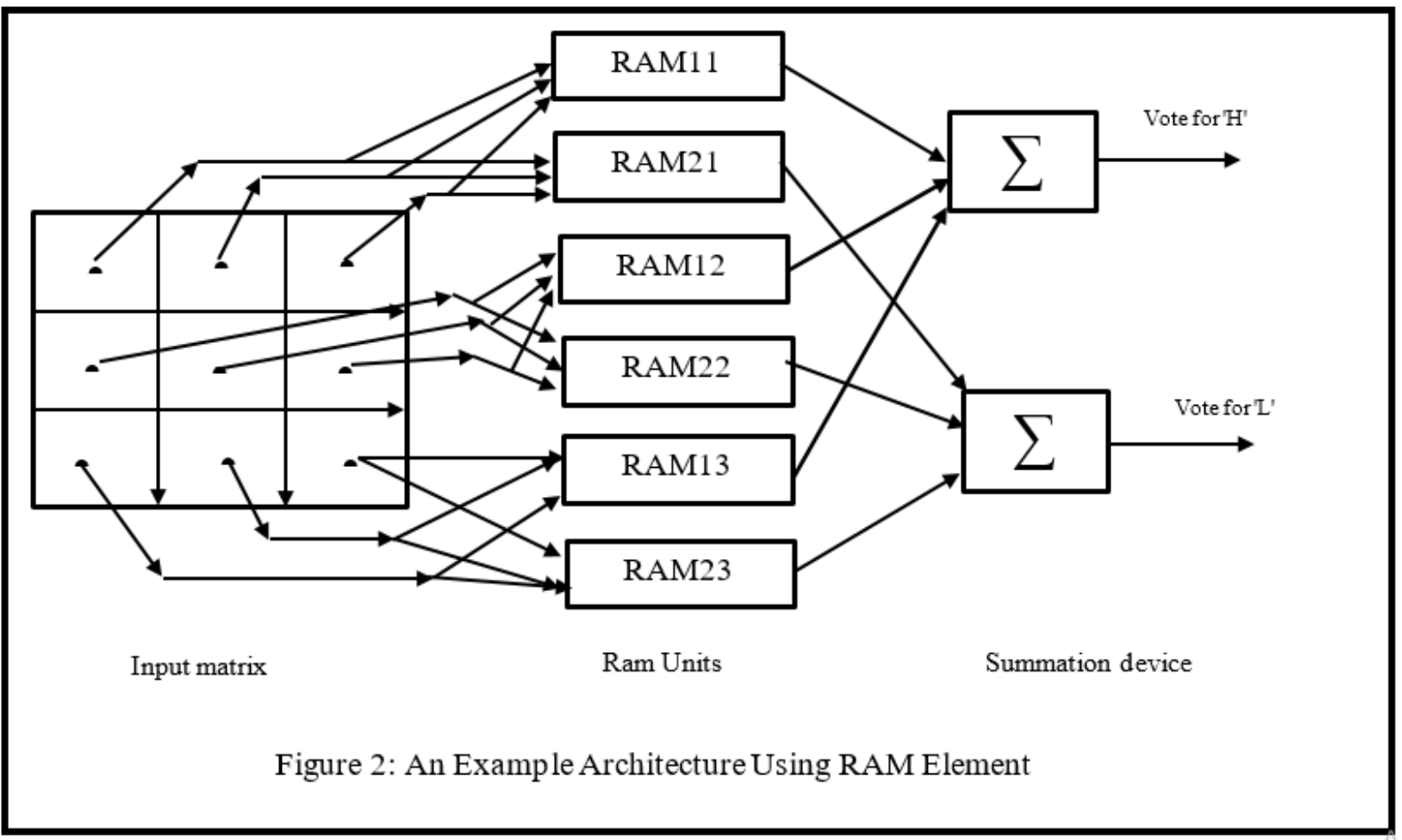

\section{Proposed Methodology}

This research suggests to modify the classification performance of $n_{\text {_ }}$ tuple method, must enhancement the characteristics of the images used. figure 3 illustrate the main steps of new technique. 


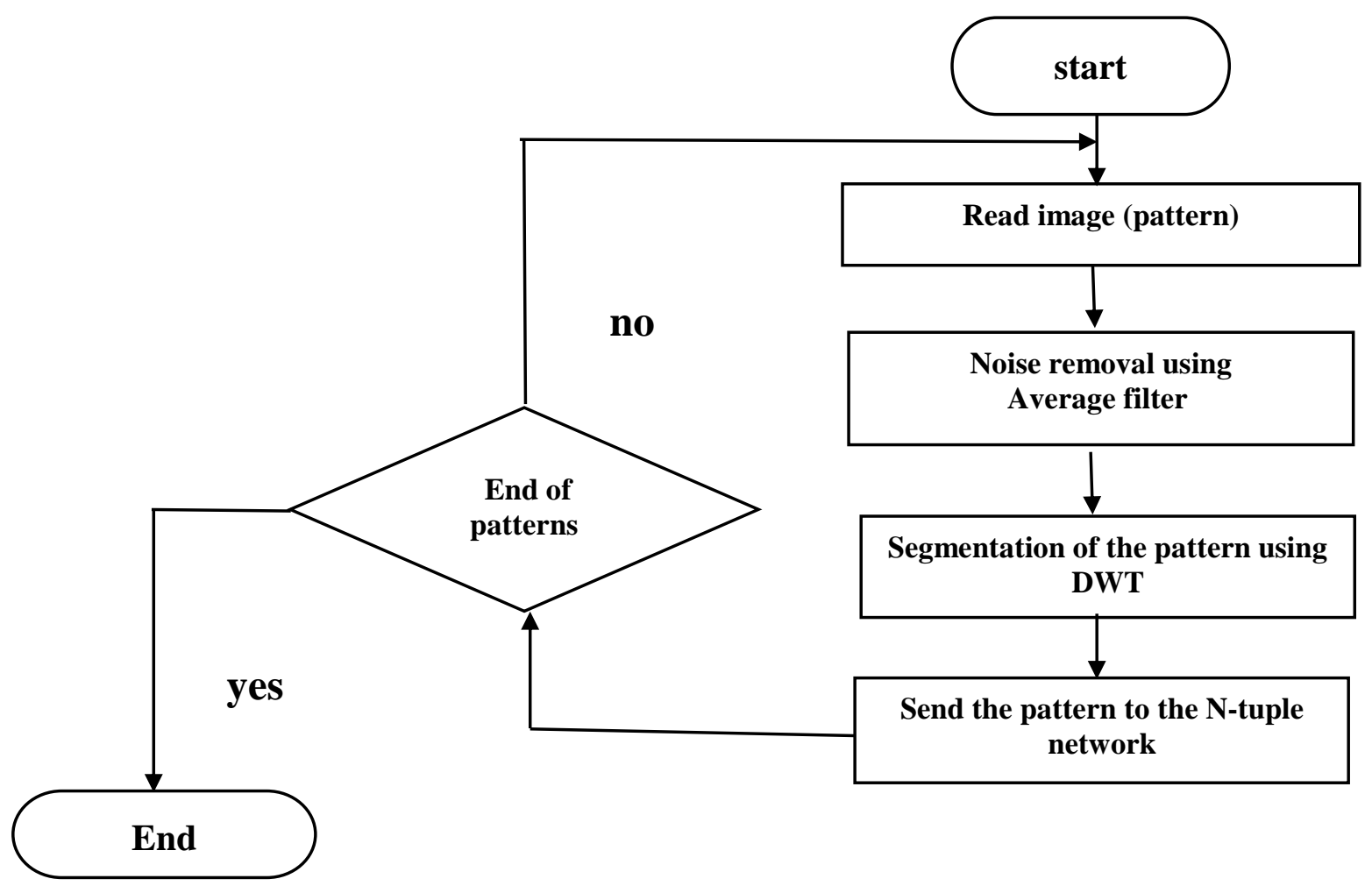

Figure 3: Block Diagram of proposed system

\subsection{Pattern Classes Used}

Every one of the investigations performed in this exploration manage written by hand characters from start to finish. The decision of transcribed characters is made because of the major thought that if profoundly precise acknowledgment of manually written characters was accomplished this would clearly end up being a best and instantly usable apparatus in manlmachine correspondence. Likewise, the method would offer degree for application to other example acknowledgment undertakings , what's more the effectiveness and optimality of execution can promptly be evaluated because of the way that the information to be perceived. All experiments were based on 80 patterns of 26 characters (A to Z) in the same pattern database. They were taken from 80 different subjects and converted by the scanner to computer into $64 \times 64$ binary patterns. The data patterns are used later for training and testing. Figure 4 shows some examples of hand written characters.
$a b c d e f g a b c d e f g a b c d e f g$
hijkl mnhijkl mnhijkl mn

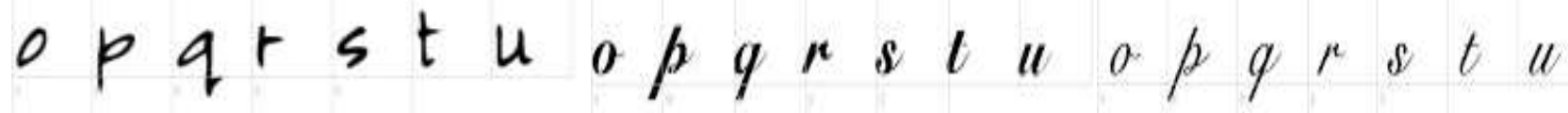
$\vee W \times \vee z \quad$ v $w x .4 z \quad$ v $w x .4 z$

Figure 4: Some examples of hand written characters 


\subsection{Remove The Noise}

Noise removing is the way toward decreasing commotion from an digital image. The primary motivation behind noise removing is to improve the quality of an image. Generally, images are debased with the noise when they are transmitted or amid image obtaining process. In the spatial area, sifting relies upon position and its neighbors. Average filter the most straightforward and the least demanding technique to actualize the smoothing of images i.e. limiting the degree of power variety among neighboring pixels. It is additionally used to limit noise in images as often as possible. The second step is separating and remove the noise from Sub Image utilizing Average Filter. In this progression the separating idea is to supplant the handling pixel component esteem in a picture grid with the normal estimation of its neighbors including itself. Over and over it expels those pixel esteems which are not speaking to their environment. Normal channel is to some degree comparable to the convolution channel. This channel additionally depends on part to speak to the shape and size of the region to be tested while mean is computed. Normal Filter peruses the information picture and sets the picture header data for the yield picture. It applies the smoothing calculation and sets the yield picture as contribution for the following emphasis if it's not the last cycle and composes the yield picture after finishing of the considerable number of emphases. The mean calculation works by including all the encompassing or neighbor pixel esteems and takes the mean or normal of those qualities. The subsequent esteem is put in the focal pixel.

\subsection{The Discrete Wavelet Transform (DWT)}

Wavelet changes are productive device for character acknowledgment. It deteriorates a picture of a character into an arrangement of various determination sub-pictures, comparing to the different recurrence groups. This outcome in space recurrence limitation which is useful for separating applicable highlights. Two dimensional discrete wavelet change (DWT) is utilized to remove the highlights, as it is very much recognized that DWT coefficients can give an effective understanding into a picture's recurrence and spatial attributes. Given a picture $\mathrm{f}(\mathrm{I}, \mathrm{j})$, its $2 \mathrm{D}$ DWT change is defined as follows:

$$
f(u, v, . .)=\sum_{i, j} f(i, j) g_{u, v, \ldots}(i, j)
$$

Where $\mathrm{i}$ and $\mathrm{j}$ are the variables of space, and $\mathrm{u}, \mathrm{v}, \ldots$ Transform domain variables. Domain variables. The wavelet features are coefficients for transforming 2-D discrete wavelets, as they can provide a clear insight into both the images' frequency and spatial properties. Each word image in our implementation is broken down by 4 wavelet transformation levels. Each image can be decomposed into four subbands at the ith level: LLi, LHi, HLi and HHi. The LLi subband talks to the low recurrence pieces, and HHi refers to the high recurrence. The LHi subband applies to the even low and vertical high recurrence components, while HLi talks about high and low recurrence components.. With a specific end goal to acquire the following level in the disintegration, the LLi sub band can be additionally deteriorated to achieve LL4, and wavelet highlights utilized as a part of our framework are extricated from the LL4 sub band. Figure 5 demonstrates the execution of DWT on each character. .

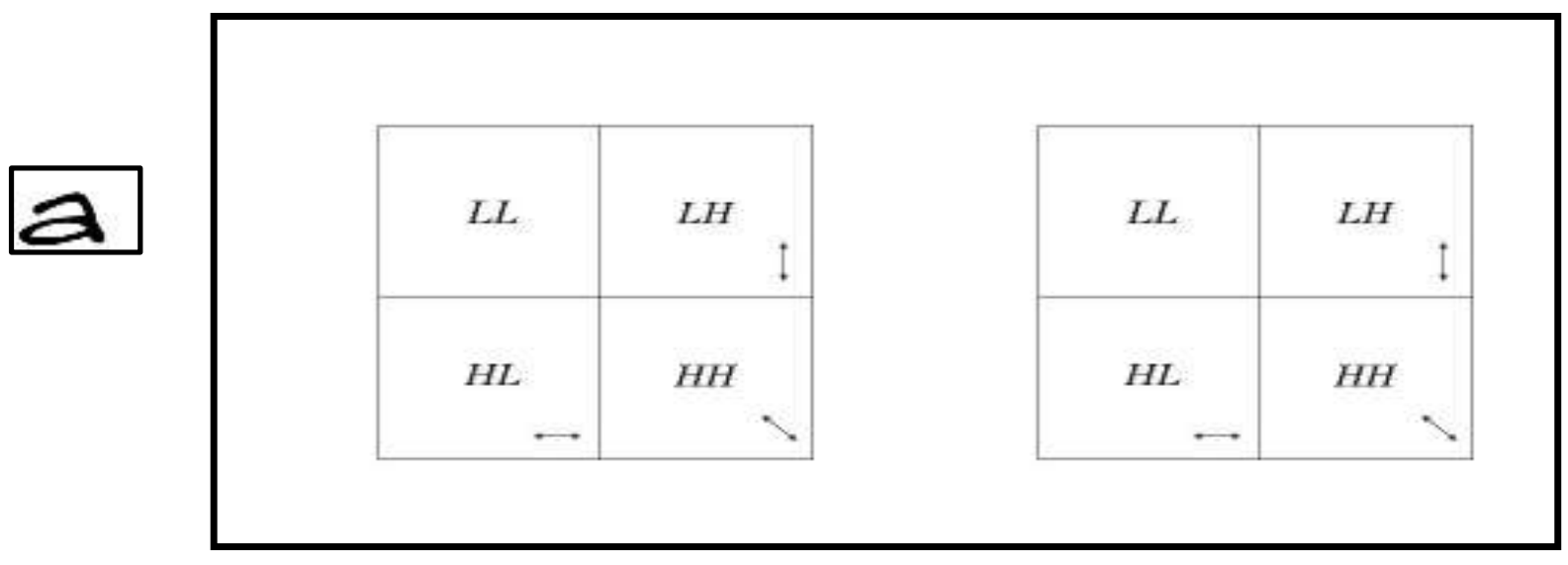

Figure 5: demonstrates the execution of DWT on each character. 
After isolating the pixels of the character image using DWT, these pixels entered to the n-tuple network which has 26 discriminators, each one to classify one character.

\section{Performance of the Proposed system}

Initially, experiments were carried out with the single layer network (simple $\mathbf{n}$-tuple scheme) in order to provide a reference point and determine the optimum characteristics which is important for the first stage of the modified system, and to measure the enhancement of the recognition level in the modified scheme. This experiment is repeated with the proposed system. The results are presented in table 1, it has been shown that, there is improvement in the performance, that because when implement the noise removal and DWT increased the system tries to cover all the probabilities to the features and gives high confidence to these features.

Table 1: Recognition with Proposed System

\begin{tabular}{|c|c|c|c|}
\hline & Correct & Reject & Error \\
\hline Original system( single layer network) & $64.8 \%$ & $35.2 \%$ & $0 \%$ \\
\hline $\begin{array}{c}\text { Modified system with just noise removal } \\
\text { Modified system with proposed system } \\
\text { (Noise removal + DWT) }\end{array}$ & $77.6 \%$ & $21.6 \%$ & $0.8 \%$ \\
\hline
\end{tabular}

From these results we noted that we could increase the recognition level from $64.8 \%$ to $84 \%$ in optimum performance. We could diminish the dismissal level of the system from $35.2 \%$ to $0.8 \%$ that primarily in light of the fact that the vast accentuating on the idea of the highlights and giving high certainty to the reactions that accomplished from the system. We note from table 1 the error rate of the network in simple digital learning network was $0 \%$.

This due to using the unconstrained patterns (hand-written numerals) in this work, which are include overlapping and big similarity among its categories. This overlapping or similarity leaded the network to rejection on some patterns (more than two discriminators recognized the pattern), therefore the error rate is $0 \%$. When increasing the confidence of responses of the all discriminators, the net becomes more capable to recognize clearly. We reached to $0.8 \%$ error rate in optimum performance.

\section{CONCLUSION}

The main objective of the reporting work was to explore a new method to improve the performance of non-deterministic data recognition through digital learning networks using n-tuple pattern recognition methods. In this data class, unregulated handwritten characters were chosen to maximize the techniques that can be used for other data types. The proposed system which used the DWT technique and noise removal of each n-tuple state, was introduced in order to increase the ability of the n-tuple recognizer for feature extraction since the information of each feature (n-tuple) will be available in each class of the recognizer to differentiate between strong features and weak ones. Therefore, proposed n-tuple scheme can be utilized to increase the decision confidence between data classes of low reparability. When implementing the proposed technique to the single layer recognizer, it could be found that this technique enhanced the correct recognition from $64.8 \%$ to $84 \%$. Beside the rejection was decreased from $35.6 \%$ to $15.2 \%$. However, the proposed method gave a higher performance and confidence.

\section{REFERENCES}

1- Albrecht S.,“ A Modular Neural Network Architecture with Additional Generalization Abilities for High Dimensional Input Vectors”,Ph.D. thesis , Manchester Metropolitan University, Department of Computing, September 1996.

2- Belanche L., Nebo A.,” INTELLIGENT DATA ANALYSIS AND DATA MINING “ 2001 .

3- Carl G. Looney, “Opto-Mechatronic Systems Handbook: Techniques and Applications”, 2002.

4- Kolcz. A, Sun.X and Kalita.J , "Efficient Handling of High-Dimensional Feature Spaces by Randomized Classifier Ensembles,", 2003 . 
5- G. Raju and K. Revathy."Wavepackets in the Recognition of Isolated Handwritten Characters", Proceedings of the World Congress on Engineering 2007 Vol I WCE 2007, July 2 - 4, 2007, London, U.K

6- R. S. Stankovic, B. J. Falkowski. "The Haar wavelet transform: its status and achievements", Computers and Electrical Engineering 29 (2003) 25- 44.

7- Chih-Chung Chang and Chih-Jen Lin, LIBSVM : "A library for support vector machines”. ACM Transactions on Intelligent Systems and Technology, 2:27:1--27:27, 2011.

8- P. S. A. Chakravarthy, A. S. N., Penmetsa V. Krishna Raja, "Handwritten Text Image Authentication Using Back Propagation," arXiv Prepr. arXiv, no. 1110.1488, 2011.

9- M. N. Abdi and M. Khemakhem, "Arabic writer identification and verification using template matching analysis of texture,” Proc. - 2012 IEEE 12th Int. Conf. Comput. Inf. Technol. CIT 2012, no. December, pp. 592-597, 2012.

10- Y. Hannad, I. Siddiqi, and M. E. Y. El Kettani, "Writer identification using texture descriptors of handwritten fragments," Expert Syst. Appl., vol. 47, pp. 14-22, 2016.

11- Y. Hannad, I. Siddiqi, Y. El Merabet, and M. El Youssfi El Kettani, “Arabic writer identification system using the histogram of oriented gradients (HOG) of handwritten fragments," in Proceedings of the Mediterranean Conference on Pattern Recognition and Artificial Intelligence, 2016, pp. 98-102.

12- H. Sheikh, A., Khotanlou, "Writer identity recognition and confirmation using persian handwritten texts," Int. J. Adv. Appl. Sci., vol. 6, no. 2, pp. 98-105, 2017.

13- J. H. AlKhateeb, F. Khelifi, J. Jiang, and S. S. Ipson, “A new approach for off-line handwritten Arabic word recognition using KNN classifier,” in 2009 IEEE International Conference on Signal and Image Processing Applications, 2009 , pp. 191-194. 